\title{
Estudio socioeconómico de los productores de banano orgánico, Cantón Milagro, Ecuador
}

\section{Socio-economic study of organic banana producers, Cantón Milagro, Ecuador}

\author{
Antonio Gonzalo Álava Murillo ${ }^{1}$, \\ Mariana del Rocío Reyes Bermeo ${ }^{2}$, Renny José Tapia Bolaño ${ }^{1}$ \\ ${ }^{1}$ Universidad Agraria del Ecuador, Guayaquil, Ecuador \\ aalava@uagraria.edu.ec, rennyjose97@outlook.es
}

${ }^{2}$ Universidad Técnica Estatal de Quevedo, Quevedo, Ecuador mreyesateq.edu.ec

\section{(c) $(1) \Theta$}

Esta obra está bajo una licencia internacional Creative Commons Atribución-NoComercial 4.0.

\section{Resumen}

Enviado: $\quad 2021 / 09 / 17$

Aceptado: $\quad 2021 / 11 / 24$

Publicado: $\quad 2021 / 12 / 30$

La producción de banano (Musa paradisiaca L.) es considerada una de las actividades agrícolas de mayor importancia económica del Ecuador, pues este sector representa una importante fuente de generación de empleo en el país. El objetivo de este trabajo de investigación fue identificar los factores socioeconómicos y tecnológicos que potencian y limitan la situación actual de los pequeños productores de banano orgánico del cantón Milagro, provincia del Guayas-Ecuador. Esta investigación de tipo descriptiva consistió en la recolección, la descripción, el registro, el análisis y la interpretación de información destinada a caracterizar la situación actual de los productores de banano orgánico en el mencionado sector. Los productores que conformaron la muestra de estudio tienen un promedio de edad de 55 años. La mayor parte de los agricultores señalan que sus ingresos provienen de la actividad bananera, y que su producción se encuentra entre 151 a 200 cajas mensuales. Entre los factores que más inciden en los costos de producción están la mano de obra, los insumos y el proceso logístico. La mayoría se financia con recursos propios. Los productores que forman parte de esta muestra poseen cultivos de 1.5 a 5 hectárea de banano de la variedad Cavendish. Para realizar las labores de mantenimiento del cultivo, cosecha y empaque utilizan mano de obra contratada y familiar.

Palabras clave: Agricultores, bananeros, factores, incremento, producción.

Sumario: Introducción, Materiales y Métodos, Resultados y Discusión y Conclusiones.

Como citar: Álava, A., Reyes, M. R. \& Tapia, R. (2021). Estudio socioeconómico de los productores de banano orgánico, Cantón Milagro, Ecuador. Revista Tecnológica - Espol, 33(3), 168-180.

http://www.rte.espol.edu.ec/index.php/tecnologica/article/view/869 


\begin{abstract}
Banana (Musa paradisiaca L.) production is considered one of the most economically important agricultural activities in Ecuador since this sector represents an important source of employment creation in the country. The objective of this research was to identify the socioeconomic and technological factors that enhance and limit the current situation of small organic banana producers in the Milagro canton of Guayas province in Ecuador. This descriptive research consisted of the collection, description, recording, analysis, and interpretation of information aimed at characterizing the situation of organic banana producers in this sector. The producers in this study sample are 55 years old as an average. Most of the farmers indicate that their income comes from the banana activity and that their production is between 151 to 200 boxes per month. Among the factors that most affect production costs are labor, supplies, and logistics. Also, most farmers are financed with their own resources. The producers in this sample own 1.5 to 5 hectares of Cavendish bananas. They use hired and family labor to carry out the maintenance and harvesting of the crop, and the packaging.
\end{abstract}

Keywords: Farmers, banana, factors, increase, production.

\title{
Introducción
}

El banano (Musa paradisiaca L.) se cultiva en abundancia en todas las regiones tropicales del mundo, de ahí la gran importancia que tiene en la economía de muchos países tropicales: África, Brasil, Colombia, China, Costa Rica, Ecuador, Filipinas, Guatemala, Honduras, India, Indonesia, Panamá, República Dominicana y Tailandia. Esta condición es la razón por la que estos países siempre están buscando estrategias y mecanismos que incrementen las exportaciones de estos productos, tales como políticas de subsidios y de nuevos impuestos, programas de incremento de la productividad y capacitaciones a productores, entre otros (Trujillo et al., 2021).

En América Latina, la productividad del banano se puede ver afectada por ciertas condiciones naturales y atmosféricas, que luego inciden negativamente en el nivel de sus exportaciones. Un ejemplo de estas condiciones climáticas adversas constituye el fenómeno de El Niño, que se produce en países de América Central y América del Sur, como Costa Rica, República Dominicana, Ecuador y Colombia. Este fenómeno provoca un gran impacto en los ecosistemas de la región: inundaciones, sequías... y trae como consecuencia niveles bajo de productividad agrícola y escases de alimentos, entre otros (Ramírez, et al., 2019).

La producción de banano en Ecuador es considerada de gran importancia económica en el sector agrícola, pues constituye una de sus principales fuentes de generación de empleo, tanto en la fase de producción como de comercialización de la fruta. Según cifras del Banco Central del Ecuador (BCE), el sector bananero aporta con un 2\% al Producto Interno Bruto (PIB) y alrededor del 35\% al PIB agropecuario, lo que lo convierte en una pieza clave para sostenibilidad de la economía ecuatoriana. Considerados estos antecedentes, se convierte en una prioridad el análisis de las condiciones de producción del banano y de todas sus modalidades. Este estudio aporta con información importante acerca de una de ellas: la producción del banano orgánico de los pequeños productores, dado que, en la actualidad, existe un crecimiento de la demanda de este tipo de banano en los mercados internacionales, lo que, a su vez, ha originado que se incremente la producción de esta fruta a nivel nacional para satisfacer dicha demanda (Villanueva et al., 2020).

Por otro lado, el aumento en el uso de plaguicidas para combatir los diferentes tipos de plagas producidos por las condiciones climáticas ha traído consigo diversos efectos negativos, entre los cuales se pueden mencionar aquellos que impactan la salud humana y el medio 
ambiente, así como aquellos que han causado estragos en la economía de los pequeños productores con el incremento en los costos de producción (García et al., 2019).

En el Ecuador, el banano es el segundo producto de exportación después del petróleo, y su venta sigue al alza. Entre los principales mercados que demandan esta fruta se pueden mencionar a la Unión Europea, Rusia, Estados Unidos, Turquía y China, entre otros. Cabe resaltar que el Ecuador se ha convertido en el mayor productor y exportador de banano del mundo, alcanzando un promedio aproximado de venta anual de seis millones de toneladas métricas (León et al., 2020).

La producción de banano orgánico en el país la realizan, generalmente, pequeños agricultores, quienes han optado por asociarse para sacar ventaja de ello, por ejemplo, en la adquisición de insumos, información y tecnología. Esto ha posibilitado asegurar la cadena de comercialización de la fruta e, incluso, ha permitido reducir sus costos de producción al compartir mano de obra. Asimismo, la creación de asociaciones y gremios han adquirido mayor fuerza para negociar en mejores condiciones la venta de su producción a los exportadores internacionales (García et al., 2012). El banano orgánico se caracteriza por no utilizar pesticidas en su cultivo, utilizando como alternativa métodos agrícolas tradicionales. Por otro lado, la demanda creciente en el mercado internacional de este tipo de productos orgánicos obliga, a quienes producen bajo esta modalidad, a obtener certificaciones que garanticen una producción libre de contaminantes. Además, es importante señalar que el buen precio que se obtiene de esta fruta está incentivando a muchos productores a incursionar en su cultivo (Gonzáles, 2018).

La producción orgánica del banano demanda la colaboración de un mayor número de trabajadores para controlar la propagación de hierba mala, pues se la realiza manualmente con herramientas como machetes o moto guadañas. De igual manera, el control de plagas y enfermedades debe realizarse con productos que no contaminan el entorno y que, por lo general, son más caros. El costo de estos insumos, así como el incremento de la mano de obra eleva el costo de producción de la fruta. Un ejemplo del incremento en el requerimiento de mano de obra se lo puede encontrar en finca La Sabana; donde laboran seis trabajadores durante la época de lluvia y cuatro trabajadores durante la época de sequía para controlar las plantas arvenses (Estrada, 2018).

Muchas familias de la costa ecuatoriana dependen de la producción agrícola del banano. Por ello, para el Gobierno nacional se ha convertido en una prioridad buscar estrategias para abrir nuevos mercados internacionales y alcanzar altos niveles de competitividad. En ese sentido, se ha impulsado la producción con altos estándares de calidad que garanticen el valor nutritivo de la fruta, que contiene macro y micronutrientes y vitaminas A, C, B1, B2, B6, B9, que, por supuesto, son de beneficio para la salud (Zhiminaicela et al., 2020).

A pesar de los esfuerzos descritos, los pequeños productores, como, por ejemplo, aquellos que conforman la Asociación Producción Agrícola "Mundo Nuevo" (ASOMUNUE) del cantón Milagro, deben afrontar la reducción del precio de la caja de banano por debajo de los costos oficiales establecidos para su venta; esto debido a las variaciones que se producen en la oferta y demanda de la fruta en los mercados internacionales. El precio de su caja varía dependiendo de la época; los tres primeros meses del año corresponden a la temporada alta de precios, en tanto que el resto del año, a la temporada baja. Durante este período, la caída de los precios no permite, en ocasiones, ni siquiera cubrir los costos de producción de la fruta. Por lo tanto, el incumplimiento del precio oficial de la caja del banano por parte de las empresas exportadoras es uno de los principales inconvenientes que deben sortear estos pequeños productores (García et al., 2016), quienes, como se ha dicho, encuentran en la organización 
gremial una estrategia para fortalecer su capacidad de negociación en el mercado. Estas asociaciones facilitan el acopio de las unidades de producción, donde se fomenta el mejoramiento de la calidad de la fruta, la reducción de costos y la implementación de sistemas de control de calidad para lograr la certificación por parte de las empresas certificadoras (Ortega et al., 2019).

En este contexto, los pequeños productores bananero están obligados a asociarse, identificar sus debilidades y analizar los factores que inciden en el funcionamiento y en la administración de su sistema de producción agrícola. Todo esto con el objetivo de alcanzar una gestión económica competitiva, optimizar los diferentes procesos para reducir costos e incrementar su productividad y su rentabilidad. Cabe señalar que, para lograr estos objetivos, el mejoramiento de los estándares de calidad en los procesos de comercialización cumple un rol estratégico también (Centenaro y Navas, 2021).

La producción de banano bajo la modalidad orgánica se ha venido incrementado en los últimos 10 años en el Ecuador. Estos sistemas de producción están encaminados al desarrollo sostenible, buscando mejorar la alimentación humana y la calidad de los suelos. También estas formas de producción han permitido el crecimiento de la economía del país. Sin embargo, los mayores problemas a los que se enfrenta el pequeño productor, además de los descritos, corresponde a las exigencias de certificación de calidad que demandan los mercados internacionales, y a los cuales muchos de estos no tienen acceso (Quezada, et al., 2021).

La producción de banano orgánico se conecta con el movimiento internacional de comercio justo, que promueve relaciones más justas y equilibrada entre productores y consumidores en el marco del desarrollo sostenible, mejores condiciones comerciales y el respeto a los derechos laborales de los trabajadores. Este trato justo está orientado sobre todo a los pequeños productores agrupados en asociaciones y comprometidos con minimizar los impactos ambientales a partir del uso de sistemas de producción orgánica (Muñiz-Jaime, et al., 2019).

Los mercados internacionales tienen gran interés por fomentar el desarrollo sostenible, demandando una producción amigable con el medio ambiente. En ese sentido, es necesario que los pequeños productores de banano orgánico se inserten en este este esquema de producción para ser más competitivos. Desde otra perspectiva, el modelo de comercio justo es una oportunidad para que los pequeños productores mejoren sus procesos de producción, y, de este modo, puedan mejorar su rentabilidad e incrementar los salarios de sus trabajadores. Además, la obtención de la certificación otorgada por este modelo de comercio justo permite el fortalecimiento de las relaciones entre productores, empresa y consumidores (Arciniega y Gonzáles, 2021).

Las certificaciones orgánicas de las unidades de producción son de gran importancia a la hora de conseguir ventajas competitivas en los mercados internacionales, que son muy exigentes en cuanto a la calidad de la fruta. De ahí que la agricultura orgánica se haya constituido en un instrumento clave para mitigar las condiciones de pobreza y mejorar las condiciones económicas de las familias de muchos productores agrícolas, sobre todo, gracias a las ventajas que brinda este modelo de producción sostenible (Federación Ecuatoriana de Exportadores [FEDEXPORT], 2018).

La gestión de producción agropecuaria tiene como finalidad la planificación, organización, dirección y control eficientes de sus recursos. De ahí que, en el marco de esta 
gestión, la producción del banano requiere establecer parámetros de control para fortalecer la eficiencia en los procesos productivos de la fruta (Vite, et al., 2020).

La presente investigación se desarrolló, por un lado, con la finalidad de encontrar estrategias y acciones que logren ampliar la asociatividad de los productores bananeros afincados en el cantón Milagro, y, por otro, proponer una estructura social, económica y tecnológica que permita acrecentar las oportunidades de crecimiento y desarrollo de la productividad en la siembra de banano orgánico. En ese contexto, las interrogantes que busca responder esta investigación están relacionadas a los factores socioeconómicos que podrían estar afectando en la competitividad del productor de banano orgánico del cantón Milagro.

\section{Localización y duración de la investigación}

\section{Materiales y Métodos}

El presente estudio se realizó en la parroquia Mariscal sucre del cantón Milagro, en la provincia del Guayas. Situada en las siguientes coordenadas: UTM: 666016,93- 9773863,6. Dicho cantón tiene una superficie total de $226.02 \mathrm{Km}$ y una altitud de $11 \mathrm{msnm}$.

\section{Figura 1}

Mapa satelital parroquia Mariscal Sucre

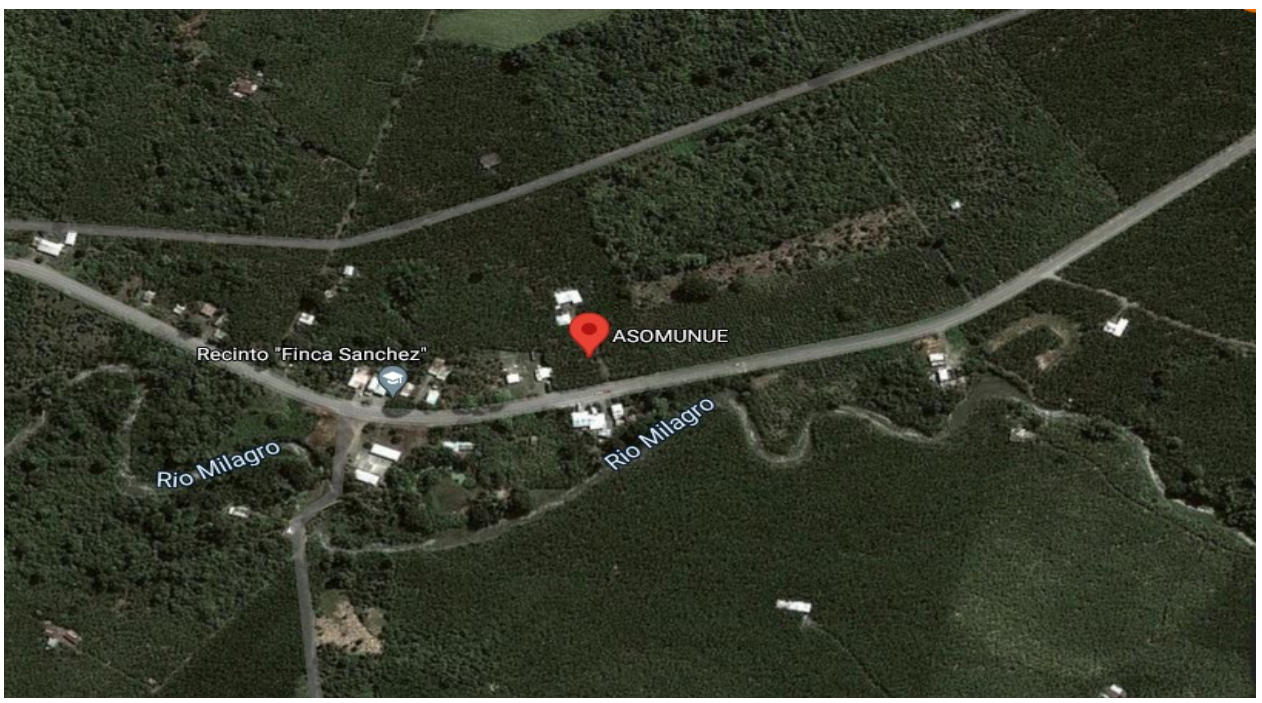

Nota. Ubicación de la asociación de los productores de banano orgánico, ASOMUNUE.

\section{Tipo de investigación}

Esta investigación de tipo descriptivo incluye un estudio de campo que se propuso recolectar, describir, registrar, analizar e interpretar información acerca de la situación actual de los productores de banano orgánico en este cantón de la provincia del Guayas. Para la obtención de los datos, se aplicó una encuesta validad para asegurar la confiabilidad de sus resultados. El diseño consistió en la elaboración de un cuestionario con preguntas dirigidas a los productores de banano orgánico.

\section{Población estudiada}

La encuesta estuvo dirigida a 50 productores de banano orgánico que forman parte de la Asociación ASOMUNE (Asociación Producción Agrícola "Mundo Nuevo"). Estos resultados fueron suficientes para concretar el objetivo de esta investigación. 


\section{Procedimiento metodológico}

Para el desarrollo de esta fase de la investigación, se procedió a validar el formulario de encuesta mediante un ensayo piloto para comprobar la efectividad de este. Después de validado el cuestionario, se realizó la aplicación de la encuesta. Luego, la información recogida se ingresó a un software estadístico SPSS y EXCEL; una vez tabulada la información, se elaboraron gráficos y se procedió a analizar los resultados bajo el modelo de la estadística descriptiva.

\section{Resultados y Discusión \\ Características socioeconómicas de los productores Aspecto social}

Los resultados de las encuestas realizadas a los productores revelan que estos tienen 55 años en promedio, según la media aritmética que se aplicó para obtener este resultado (Figura 2). En relación con el nivel de instrucción, se obtuvieron los siguientes resultados: $42 \%$ de los encuestados tienen estudios secundarios, en tanto que un $40 \%$ cuenta con estudios primarios; mientras que un $6 \%$ tiene estudios de tercer nivel en la rama de ingeniería agronómica. Por el contrario, el $12 \%$ no ha cursado ningún grado de escolaridad. En un estudio similar a este realizado por Álvarez et al. (2020), en la zona norte de la provincia de Los Ríos, un 59\% de los encuestados tiene una escolaridad de secundaria completa, un $38 \%$, con grado de escolaridad superior. Por otro lado, las características socioeconómicas de los productores de musáceas en otros sectores del país indican que, en la gran mayoría de los hogares, el hombre es el que asume el rol de jefe de familia. Sus edades oscilan entre los 50 y 65 años. El nivel de educación del productor en su mayoría llega a la primaria. Sus ingresos provienen en gran medida de lo que producen sus fincas (Lara et al., 2021).

\section{Figura 2}

\section{Nivel de educación de los productores}

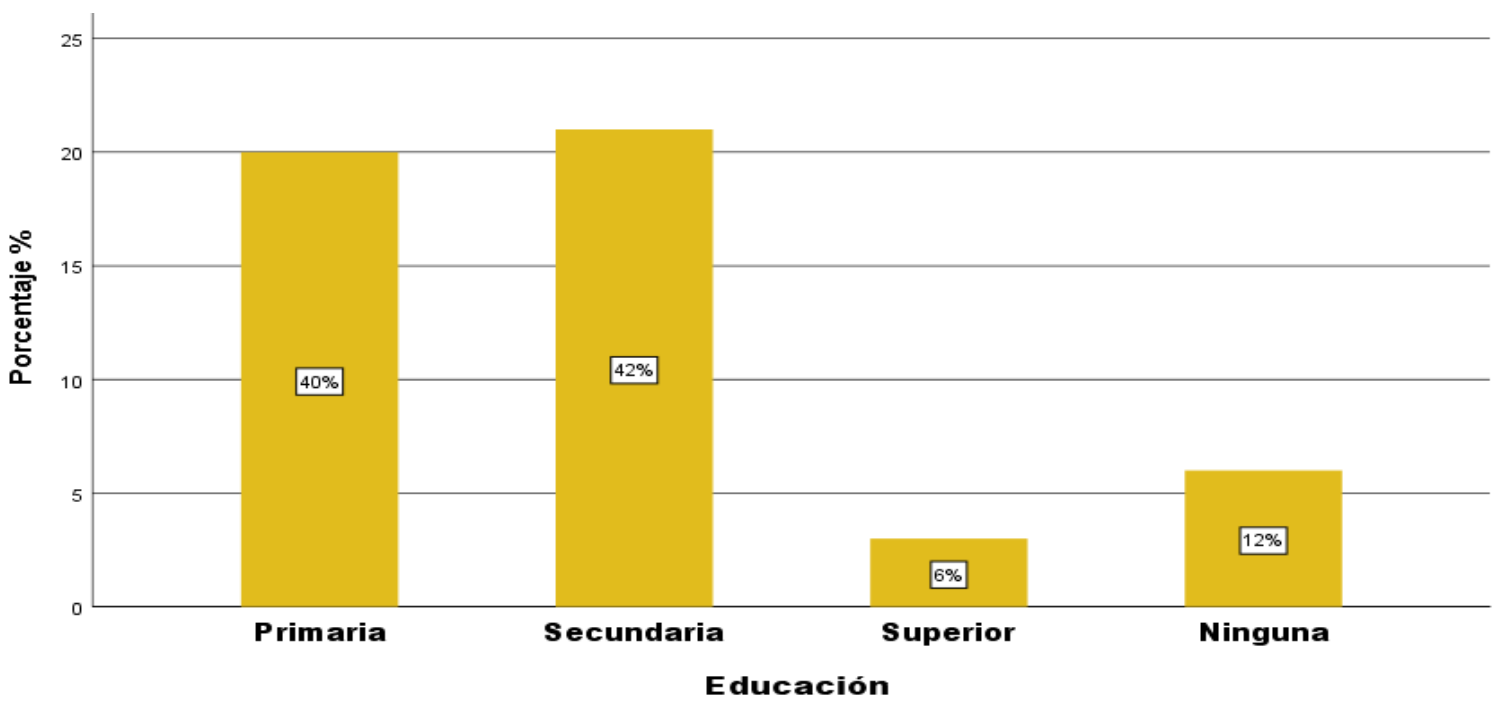

Nota. Datos sobre el nivel de estudios de los productores de banano orgánico de la zona de Milagro

\section{Aspectos económicos}

Este estudio evidenció que $56 \%$ de los productores bananeros de esta zona del cantón Milagro dependen de su activad agrícola. La mayoría de estos dedican entre 8 y 10 horas a sus actividades productivas; Por otro lado, el 26\% indica que sus ingresos económicos, sustento de sus familias, proceden de la actividad bananera y de otras actividades; un 14\% registró que sus ingresos provienen de la actividad bananera y también de los sueldos de otros familiares; solo 
el $4 \%$ señaló que sus ingresos provienen del banano y de otros cultivos agrícolas que tienen en sus unidades de producción. El 85\% de la producción bananera del país se encuentra en manos de la economía familiar campesina, sin embargo, debido a la estructura agraria de este sector, se produce la transferencia del valor generado por la EFC a empresas exportadoras de la fruta, que obtienen un mayor beneficio; estas, por su lado, ceden una parte del beneficio extra que han ganado a las comercializadoras de banano de los países importadores (Borja, 2016). En el Ecuador, la actividad bananera se ha constituido en uno de los principales rubros que dinamiza la economía del país. En tal sentido, hoy en día, existen muchas familias que dependen de esta actividad económica (León et al., 2020).

\section{Figura 3}

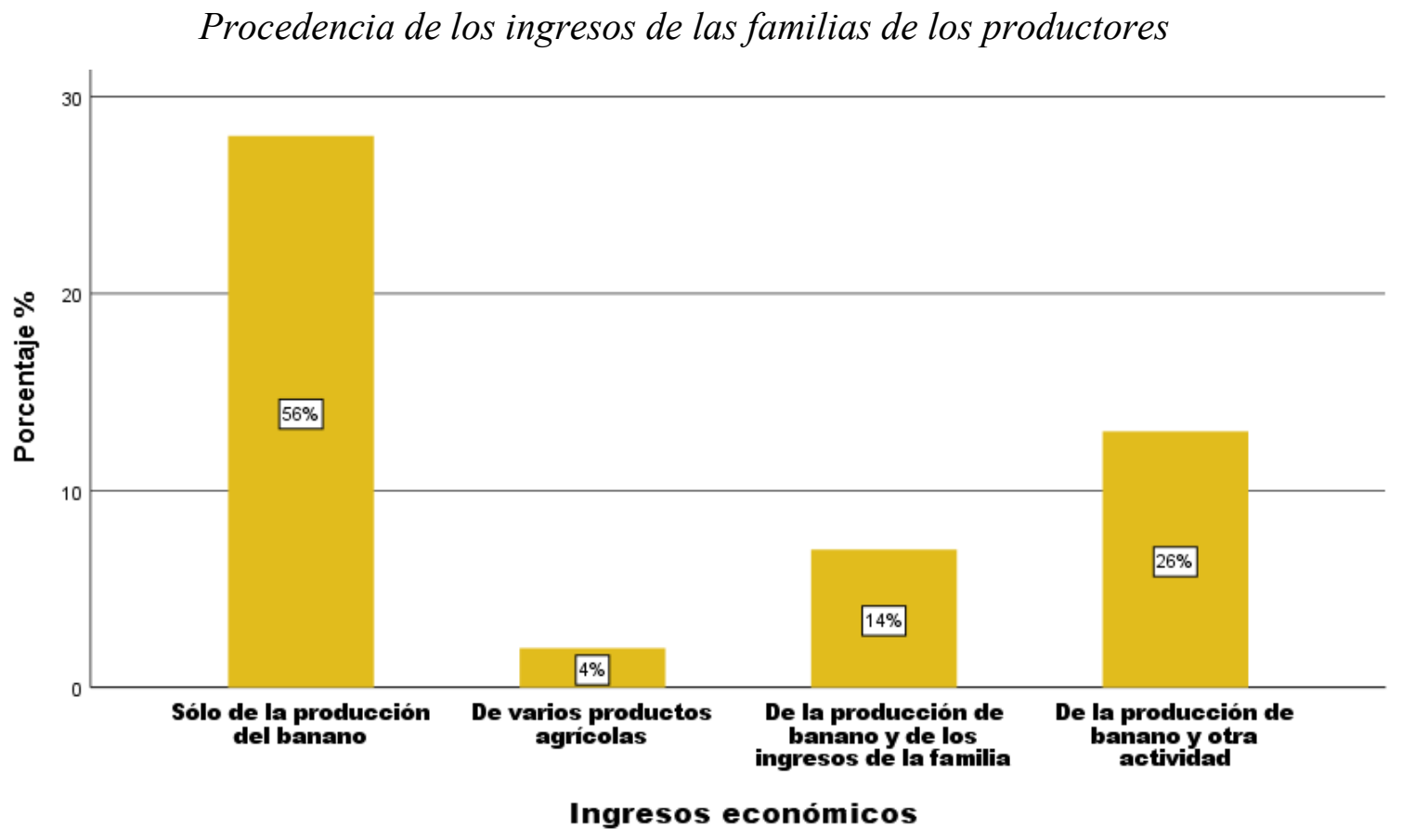

Nota. Origen de los ingresos de los productores de banano orgánico en la zona del cantón Milagro.

A partir de los datos recabados para este estudio, se pudo determinar que los productores venden entre 151 a 200 cajas de bananos a la semana, unas 2000 a 3000 cajas al año, a un precio que varía de 5 a 10 dólares la caja; la ratio promedio es de 1.2, aproximadamente. Además, se verificó que existen ciertos factores que afectan los costos de producción del banano, como se aprecia en la Figura 4. El 64\% de los productores coincide en que el costo de la mano de obra es el factor que más incide en el alto costo de producción; el $34 \%$ identificó el precio de los insumos, tales como de abonos o fertilizantes para el control de plagas y enfermedades, lo que encarece el proceso; solo un $2 \%$ considera que los costos de tipo logístico inciden más. Estos gastos solo pueden ser cubiertos con los ingresos que genera la exportación de la futa (Lalangui et al., 2018). Los agricultores bananeros ponen especial interés en el precio de la caja de la fruta a la hora de firmar un contrato de venta con la exportadora (Pardo et al., 2020). En todo proceso productivo existe la generación de costos, y la actividad agrícola no es la excepción; Dadas las dificultades, es imperativo el uso eficiente de los recursos. Para ello, es necesario implementar una adecuada planificación y un correcto registro contable que faciliten la toma de decisiones (Mora, et al., 2017). Los costos de producción en el cultivo de banano proporcionan información para la planificación de todas las operaciones que se realizan en esta actividad productiva. La producción orgánica requiere mayor inversión que la producción convencional, debido, primero, a la necesidad de mayor mano de obra por 
hectárea, además del incremento en las actividades de postcosecha y en la cadena de valor de la fruta (Mata Anchundia et al., 2021).

\section{Figura 4}

Factores que inciden en los costos de producción

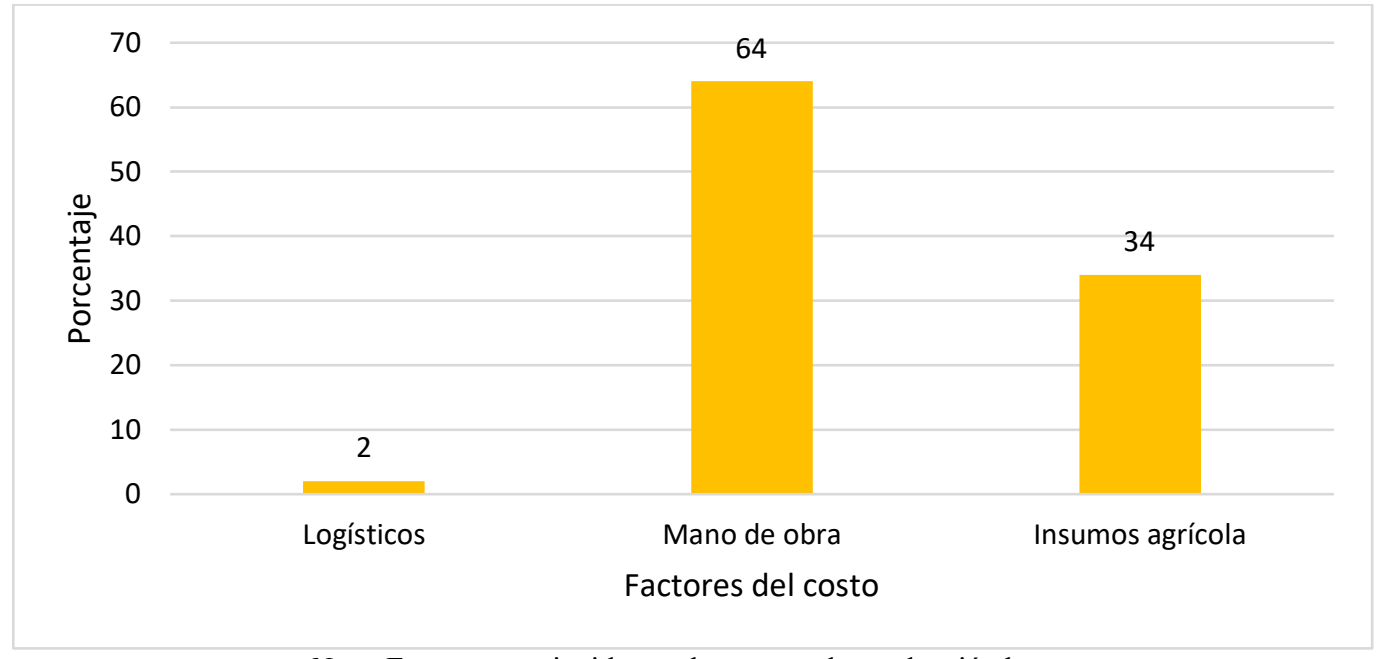

Nota. Factores que inciden en los costos de producción bananera.

En la Figura 5, se muestra que un 78\% de los productores bananeros se financia la actividad agrícola con sus propios recursos; solo un $8 \%$ lo hace con apoyo de la asociación ASOMUNUE; asimismo, un $8 \%$ financia la producción con créditos contraídos con terceras personas; mientras que el $6 \%$ de los productores mencionó que sacó un crédito a la banca. Según este estudio, todos los productores, en algún momento, han solicitado un crédito bancario para realizar mejoras en sus cultivos y la mayor parte de los productores están dispuestos a tomar un crédito para expandir su actividad (Álvarez et al., 2020). Sin embargo, el crédito productivo agrícola, sea público o privado, todavía no alcanza los niveles de otras fuentes de financiamiento. Cabe señalar que, si se compara el volumen de crédito otorgado por las instituciones financieras, es notorio que las instituciones privadas son las que en su mayoría financian al sector agrícola (Chagerben, et al., 2019).

\section{Figura 5}

Financiamiento de las actividades en el cultivo

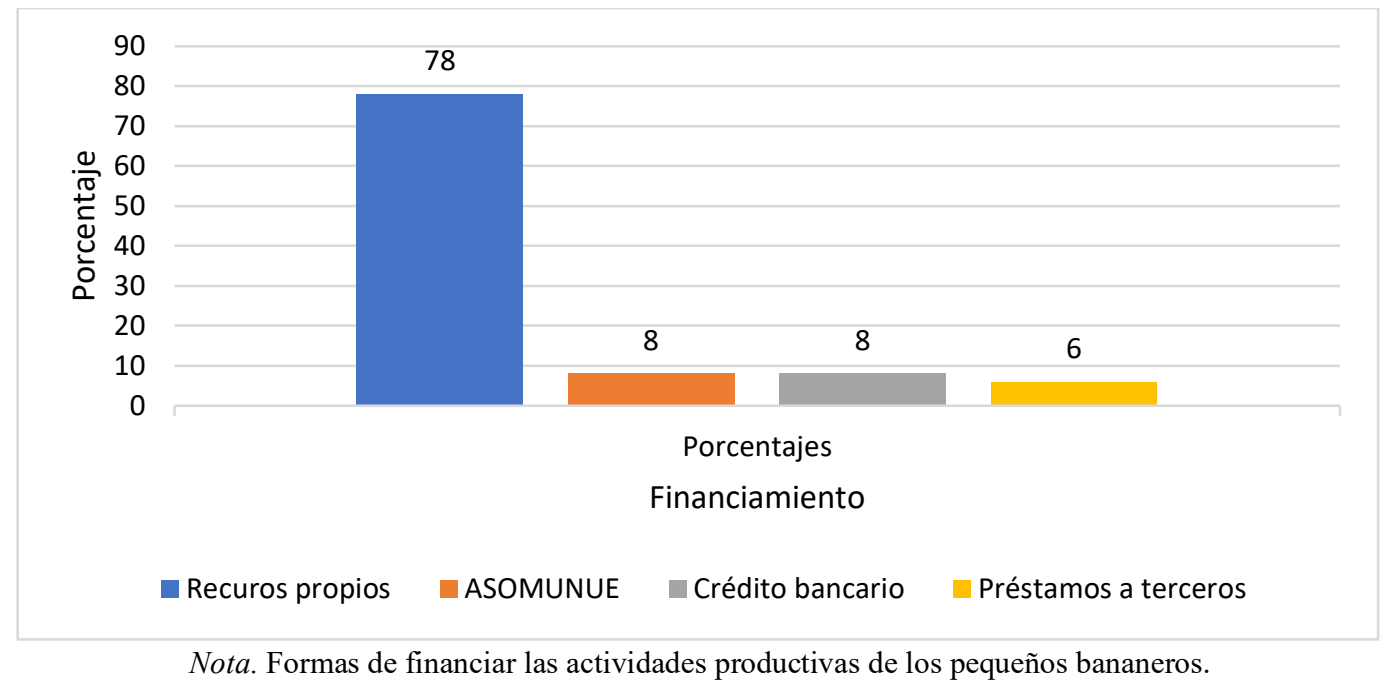

Nota. Formas de financiar las actividades productivas de los pequeños bananeros. 


\section{Aspecto productivo}

La investigación mostró que el $42 \%$ de los productores bananeros de esta zona del cantón Milagro disponen de una superficie de siembra de 2.6 a 3.5 hectáreas; en tanto que un $34 \%$ indicó que cultivan entre 1.5 a 2.5 hectáreas, y un $24 \%$, entre 3.6 a 5 hectáreas. Cabe señalar que todos tienen legalizada la tenencia de la tierra y llevan entre 10 a 20 años realizando esta actividad. La mayor parte de la producción nacional ya sea orgánico o convencional corresponde a los pequeños y medianos productores, quienes la venden a empresas comercializadoras para su posterior exportación. Este circuito ha permitido generar recursos para el sector y dinamizar la economía (León, Arcaya et al., 2020). El Programa de Desarrollo de la Productividad de los Pequeños Bananeros, mencionado en la sección anterior, brinda asistencia técnica orientada a incentivar el uso de abono orgánico entre los pequeños agricultores, producido en las propias fincas bananeras. La idea es, al mismo tiempo, aportar al cuidado del ambiente y de la salud de los trabajadores del sector y de los pobladores de las zonas aledañas (Ministerio de Comercio Exterior, 2017).

\section{Figura 6}

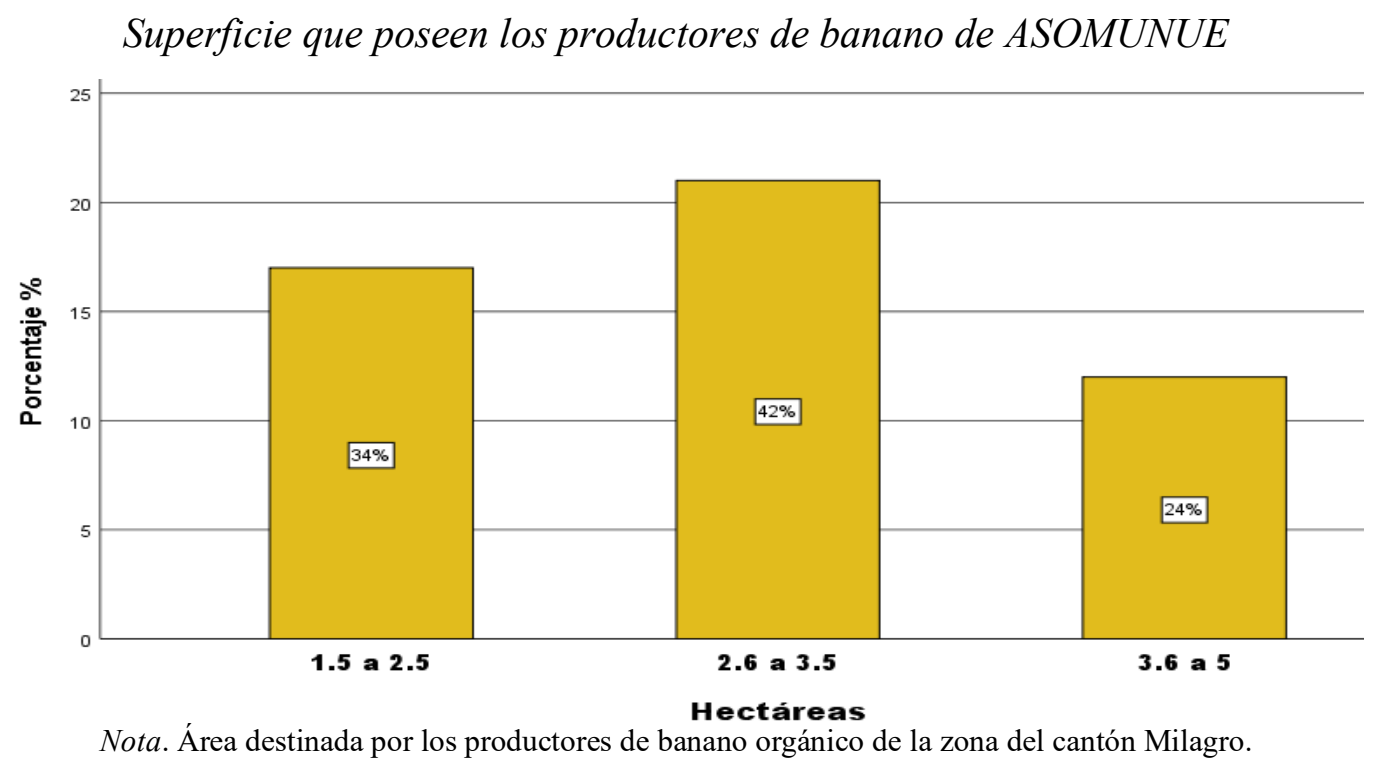

En el presente estudio se pudo evidenciar que los productores siembran la variedad de banano Cavendish, una de las más comercializadas a nivel mundial y muy resistente a plagas y enfermedades. Además, cabe señalar que el estudio mostró que la técnica de siembra de tres bolillos es la más utilizada entre los agricultores, pues este método permite una mayor penetración de rayos solares, lo que genera beneficios para la planta. La mayor parte de los productores $(82 \%)$ contrata mano de obra externa para el mantenimiento del cultivo, la cosecha y el empaque. Por su lado, el 18\% de los productores mencionaron que utilizan mano de obra familiar y contratada para cumplir con las actividades agrícolas.

En el país, la Cavendish Valery es una de las variedades más sembradas de banano con fines de exportación, debido a su gran resistencia a las plagas y a las enfermedades, a su gran rendimiento y a su perdurabilidad para no deteriorarse en el transporte (García et al., 2016). El requerimiento de mano de obra es vista como una medida positiva para garantizar el empleo de trabajadores y de las familias que se dedican a este tipo de producción en el sector (Capa et al., 2016). La mayoría de los trabajadores que están vinculados a la actividad bananera pertenecen a edades entre 40 a 69 años. La gestión laboral tiene identificada diferentes tipos de flexibilización o modos de contratación, despido, salarios, imposición de tareas, etc. El proceso productivo del banano está compuesto por cuatro fases: la siembra, el mantenimiento del 
cultivo, la cosecha y el empaque. En cada componente se realizan varias actividades que permiten seccionar el trabajo e incrementar la efectividad de este (Vitali, 2017).

De acuerdo con lo indicado por los productores de banano, las capacitaciones realizadas por la asociación ASOMUNUE les ha permitido mejorar su cultivo. Además, se muestran interesados en seguirse capacitando, como se aprecia en la Figura 7. Un 54\% de encuestados está interesado en conocer el manejo del cultivo; en tanto que un 32\% considera importante saber cómo controlar la sigatoka negra; asimismo, hay interés en aprender sobre el manejo orgánico de los cultivos y sobre los trámites para lograr una certificación orgánica; solo un $6 \%$ quiere aprender a realizar de manera eficiente la fertilización del cultivo. Los productores requieren de modo permanente planes de capacitación sobre el uso de alternativas para la fumigación de las plantaciones de banano que cumplan con las normas ambientales permitidas. Esto con el fin de precautelar la integridad física de los trabajadores y sus familias (León, 2017). La capacitación a los agricultores ecuatorianos es imprescindible para mejorar el componente tecnológico y el proceso de administración de la fase productiva, de tal forma que les posibilite ser más competitivos, y, así, incrementar sus ingresos (Intriago, 2019). Los proyectos de capacitación permiten reunir a los productores y recibir información variada sobre el tema tecnológico y la eficacia sobre el manejo de cultivo (Vallejo et al., 2016).

Figura 7

Principal interés por recibir capacitación

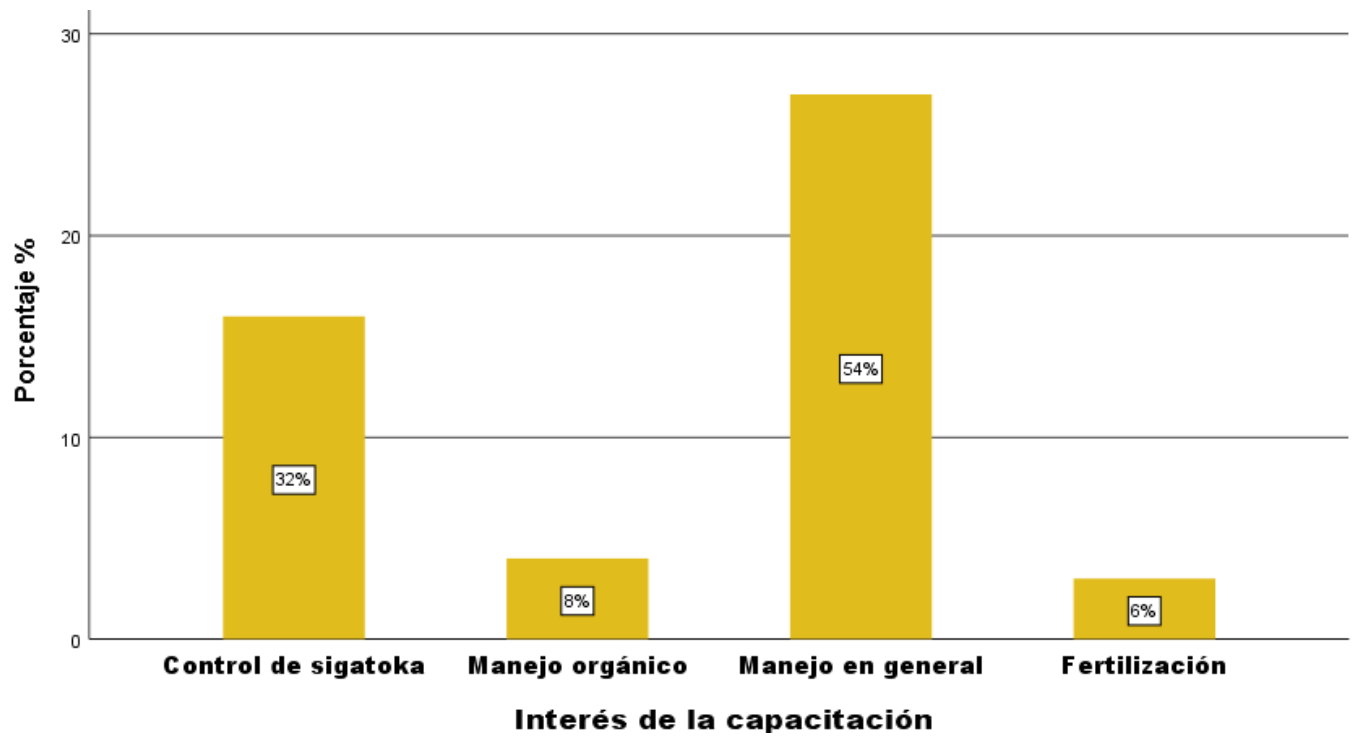

La mayoría de los productores (96\%) utiliza agua de pozo para regar el cultivo; un 4\% dice utilizar agua de rio, lo que es considerado una ventaja. El sistema de riego más utilizado es el de aspersión, el cual ha evolucionado mucho en los últimos años, hasta alcanzar un mejor uso del agua, lo que, a su vez, ha permitido contar con una menor demanda de mano de obra $\mathrm{y}$, por tanto, ha sido un procedimiento para abaratar costos (Caicedo et al., 2015). El agua es muy importante para la absorción de nutrientes del suelo. Una adecuada absorción ocasiona el normal crecimiento y desarrollo de la planta. Los requerimientos de nutrientes de la planta de banano son altos; la mayor parte de su peso es agua, es decir, entre un $85 \%$ a $88 \%$, debido a su naturaleza herbácea (Belalcazar, 1991; Velásquez, 2016). La calidad del agua de riego puede causar un impacto en los niveles de producción del cultivo, es decir, una mala calidad de agua puede causar reducciones en la infiltración afectando la productividad (Santacruz y Santacruz, 2020). 
En cuanto al manejo de los residuos plásticos, se determinó que el 58\% de los productores tienen la cultura de clasificar en bolsas separadas los envases plásticos de los residuos orgánicos. En cambio, un $42 \%$ mencionó que no los clasifican y lo botan en la basura común. La producción agrícola está relacionada con el uso de plaguicidas, lo cual genera residuos peligrosos para el ecosistema, ocasionando un impacto negativo en el ambiente y en la salud humana (Camargo et al., 2021). Es necesario el establecimiento de un plan de gestión ambiental si se quiere una empresa sostenible y socialmente responsable en el manejo de residuos. Para esto, se debe impartir capacitaciones a los trabajadores y a todas las personas que estén involucradas en las actividades productivas (García et al., 2017).

\section{Conclusiones}

Entre las principales conclusiones que se pudieron obtener está los ingresos de los agricultores que provienen, principalmente, de la actividad bananera, y cuya producción estimada es de 150 a 200 cajas semanales. Los factores que inciden más en los costos de producción del banano están la mano de obra, el precio de los insumos y el transporte. Gastos que reducen el margen de utilidad para los pequeños productores de banano orgánico.

La asociación de productores ASOMUNUE está conformada por agricultores que poseen de 1.5 a 5 hectárea de cultivo del banano de la variedad Cavendish. La mano de obra que utilizan para las labores de mantenimiento del cultivo, la cosecha y el empaque es contratada y familiar.

Se evidenció que la mayor parte de los productores utilizan agua de pozo para regar el cultivo, y el sistema de riego por aspersión es el más utilizado en la zona.

\section{Reconocimientos}

Los autores agradecen a la Asociación de Producción Agrícola "Nuevo Mundo" (ASOMUNUE), a su presidente, el Ing. Luis Angulo Calle, por su gran apoyo brindado para la realización de este trabajo de investigación.

\section{Referencias}

Álvarez, E., León, S., Sánchez M., y Cusme, B. (2020). Evaluación socioeconómica de la producción de plátano en la zona norte de la Provincia de los Ríos. Journal of Business and Entrepreneurial Studie, 4(2). https://doi.org/10.37956/jbes.v4i2.78

Arciniega, J., Gonzáles M. (2021). Prácticas organizacionales en la certificación de Comercio Justo como factor diferenciador en la oferta exportable. Revista electrónica Yura: Relaciones internacionales. 17-34

Borja, J. (2016). La producción de banano bajo el sistema de comercio justo: Un análisis del caso ecuatoriano. Siembra, 3(1), 7-10. https://dialnet.unirioja.es/servlet/articulo?codigo=6140329

Caicedo, O, Balmaseda, C, y Proaño, J. (2015). Evaluación hidráulica del riego por aspersión subfoliar en banano (Musa paradisiaca) en la finca San José 2, provincia Los Ríos, Ecuador. Revista Ciencias Técnicas Agropecuarias, 24(1), 38-43. http://scielo.sld.cu/scielo.php?script=sci_arttext\&pid=S2071

Camargo, Y., Tovar, F., Álvarez, E. (2021). Residuos de plaguicidas en cultivos del municipio zona bananera, departamento Del Magdalena, Colombia. Revista Internacional Contaminación Ambiental. 37, 145153 https://doi.org/10.20937/RICA.53725

Capa, L., Alaña, T., y Benítez, R. (2016). Importancia de la producción de banano orgánico. Caso: provincia El Oro, Ecuador. Universidad y Sociedad [seriada en línea], 8(3).pp. 64 -71. http://rus.ucf.edu.cu/

Centenaro, P., y Nava, J. (2021). Nudos críticos de procesos gerenciales en unidades productivas de banano, Milagro, Ecuador. Revista CEA, 7 (13), 1-16. https://doi.org/10.22430/24223182.1554 
Chagerben, L., Moreno, N., y Chagerben W. (2019). El crédito productivo y su incidencia en la producción agrícola del Ecuador. Revista internacional de administración Estudio de la Gestión. DOI: https://doi.org/10.32719/25506641.2019.6.1

Estrada, S., y Encalada, N. (2018). Producción de banano orgánico, una experiencia exitosa en La Sabana del Cantón Pasaje, Provincia El Oro, Ecuador. Revista Cientifica Agroecosistemas, 5(1-Ext), 21-27. http://aes.ucf.edu.cu/index.php/aes/index

Federación Ecuatoriana de Exportadores (FEDEXPORT). (2018). Reporte mensual de comercio exterior.

García Batista, R. C., Machado López, L., \& Minuche, J. L. (2017). Plan de gestión ambiental de desechos sólidos en la Empresa productora de banano, Herederos Coronel, del Cantón Machala, Ecuador. Universidad y Sociedad [seriada en línea], 9(1), pp. 100-105. http://rus.ucf.edu.cu/

García, J., Marcillo, A., y Palacios, C. (2019). Amenazas de las manchas foliares de Sigatoka (Mycosphaerella spp.) en la producción sostenible de banano en el Ecuador. Revista Verde de Agroecología e Desenvolvimiento Sustentável 14(5), 591.596.

García, L., Santillán, E., Blancas, N., y Valenzuela, P. (2012). Proyecto de inversión para el desarrollo de la producción de banano orgánico ecuatoriano y su exportación a Hamburgo-Alemania. Guayaquil: Publicaciones ESPOL.

García, M., Juca, F., y Juca. M. (2016). Estudio de los eslabones de la cadena de valor del banano en la provincia de El Oro. Universidad y Sociedad [seriada en línea], 8(3). pp. 51- 57. http://rus.ucf.edu.cu/

García Saltos, M. B., Juca Maldonado, F., \& Juca Maldonado, O. M. (2016). Estudio de los eslabones de la cadena de valor del banano en la provincia de El Oro. Universidad y Sociedad [seriada en línea], 8(3). pp. 51- 57. http://rus.ucf.edu.cu/

González, A. (2018). Prácticas ambientales y competitividad de las PYMES bananeras del cantón Machala, provincia El Oro, Ecuador. Revista Dilemas Contemporáneos: Educación, Política y Valores. http://www.dilemascontemporaneoseducacionpoliticayvalores.com/

Intriago, F. (2019). La mecanización agrícola y su impacto en el desarrollo agropecuario del Ecuador. Sathiti:sembrador, 14(2), 290-300. https://doi.org/10.32645/13906925.910

Lalangui, M., Eras, R., y Burgos, J. (2018). Costos de producción: Estimación y proyección de ingresos. http://repositorio.utmachala.edu.ec/handle/48000/12483

Lara, S., Vera, D., Cabanilla, M., y Gonzáles B. (2021). Desarrollo comunitario: Producción de Musáceas en dos zonas de la costa ecuatoriana. Revista de Ciencias Sociales (Ve), 27(3), pp. 340-354, 2021. Universidad del Zulia

León, L, (2017). La sostenibilidad ambiental en el sector productivo bananero del cantón Machala. Conference Proceedings UTMACH, 1(1). https://investigacion.utmachala.edu.ec/proceedings/index.php/utmach

León, L., Arcaya, M., Barbotó, N., y Bermeo, Y. (2020). Ecuador: Análisis comparativo de las Exportaciones de banano orgánico y convencional e incidencia en la Balanza Comercial, 2018. Revista Científica y Tecnológica UPSE, 7(2), 38-46. DOI: 10.26423/rctu.v7i2.521.

León., L, Baquero., E, y Villa., J. (2020). Incidencia de los Ingresos en la producción de banano, cantón Santa Rosa. Revista de Investigación Enlace Universitario, 19(2), 51-59

León Serrano, L.; Arcaya Sisalima, M.; Barbotó Velásquez, N.; Bermeo Pineda, Y. (2020). Ecuador: Análisis comparativo de las Exportaciones de banano orgánico y convencional e incidencia en la Balanza Comercial, 2018. Revista Científica y Tecnológica UPSE, 7(2), 38-46. DOI: 10.26423/rctu.v7i2.521

Mata Anchundia, D. Suatunce Cunuhay, J. P. \& Poveda Morán, R. (2021). Análisis económico del banano orgánico y convencional en la provincia Los Ríos, Ecuador. Avances, 23(4), 419-430.

Ministerio de Comercio Exterior. (2017). Informe sector bananero ecuatoriano. Recuperado de https://www.produccion.gob.ec/wp-content/uploads/2019/06/Informe-sector-bananeroespa\%C3\%B1ol-04dic17.pdf

Mora, J., Hullcapi, M., Escobar, D., Naranjo, D., Castro, G. (2017). Impacto de los costos de mano de obra en la gestión financiera de la producción de banano. Revista Observatorio de la Economía Latinoamericana, Ecuador.

Muñiz-Jaime, L., Vinueza-Ramírez, M., Ayón-Ponce, G. (2019). El comercio justo como alternativa para desarrollar estrategias en beneficio de los productores. Revista Científica Dominio de las Ciencias. 5(3), 589-602.

Ortega, M., Noroña, J., y Noroña, C. (2019). Diagnóstico situacional de los pequeños productores del banano orgánico de la provincia de El Oro hacia el mercado europeo. Espirales Revista Multidisciplinaria de Investigación.

Pardo, G., Narváez, C., y Erazo, J. (2020). Análisis del impacto tributario y contable por las variaciones del precio de la caja de banano en los productores del cantón Machala, Ecuador. Revista Científica Dominio de las Ciencias, 6(1), 396-428. 
Quezada Veliz, R. X., Carvajal Romero, H., \& Barrezueta Unda, S. (2021). Impacto económico de la producción bananera en el Ecuador en el periodo 2008-2016. Revista Metropolitana de Ciencias Aplicadas, 4(2), 148-157.

Ramírez, L. M., Romero, A. E., Alava, A. R., \& Jaramillo, F. (2019). Sequías: Efecto sobre los recursos naturales y el desarrollo sostenible. Revista Cientifica Agroecosistemas, 7(3), 154-162.

Santacruz, G., Santacruz, E. (2020). Evaluación del desempeño del riego por aspersión en lotes con cultivo de banana en Chiapas, México. Revista Siembra. 7(2), 001-013. DOI: https://doi.org/10.29166/siembra.v7i2.1712

Trujillo, D., Pereira, S., y Torres, G. (2021). Factores que afectan la variación de los ingresos FOB por exportación de banano y plátano ecuatoriano. Revista Economía y Negocios UTE. 12(1)1, 1-12.

Vallejo, Y., Pérez, T., Del Pozo, E., Arozarena, N., López, A. (2016). La capacitación agraria desde la visión del agricultor, en el municipio Boyeros, La Habana, Cuba. Revista Cultivos Tropicales 37(2), 149-154.

Velásquez Rodríguez, P. (2016). Implementación de un sistema productivo de banano (Musa acuminata AAA) como alternativa sustentable y competitiva para pequeños productores del municipio de AndesAntioquia. Retrieved from https://ciencia.lasalle.edu.co/ingenieria_agronomica/43

Villanueva, V., Añazco, C., y Bonisoli, L. (2020). Introducción de marca de banano orgánico en el mercado ecuatoriano. INNOVA Research Journal, 5(1), 166-183. https://doi.org/10.33890/innova.v5.n1.2020.1150

Vitali, S. (2017). Precariedad en las condiciones de trabajo y salud de los trabajadores del sector bananero del Ecuador. Revista Salud de los Trabajadores. 25(1), 9-22.

Vite Cevallos, H., Townsend Valencia, J., \& Carvajal Romero, H. (2020). Big Data e Internet de las Cosas en la producción de banano orgánico. Revista Universidad y Sociedad, 12(4), 192-200.

Zhiminaicela Cabrera, J. B., Quevedo Guerrero, J. N., \& García Batista, R. M. (2020). La producción de banano en la Provincial de El Oro y su impacto en la agrobiodiversidad. Revista Metropolitana de Ciencias Aplicadas, 3(3), 189-195. 